\title{
An Inductively Coupled Plasma-Time-of-Flight Mass Spectrometer for Elemental Analysis. Part II: Direct Current Quadrupole Lens System for Improved Performance
}

\author{
D. P. Myers, G. Li, P. P. Mahoney, and G. M. Hieftje \\ Department of Chemistry, Indiana University, Bloomington, Indiana, USA
}

\begin{abstract}
An electrostatic quadrupole lens has been substituted for a cylindrical lens system used in the original inductively coupled plasma-time-of-flight mass spectrometer (ICP-TOFMS). With an improved vacuum system also installed, the cylindrical and quadrupole lenses are compared to each other and to the performance of the prototype ICP-TOFMS. The quadrupole lens requires no tradeoff between ion throughput and resolving power as was encountered with cylindrical lenses. The background noise in both ion-optical systems is within the same order of magnitude. Images of the ion beam formed by each ion-optical system have been obtained on a microchannel plate-phosphor screen. The quadrupole lens shows a higher ion-beam flux and produces a slitlike focus required in the orthogonal ICP-TOFMS instrument. Signal-to-noise ratios in the ICP-TOFMS can be improved by using a technique called pulsed-ion injection that is particularly convenient with the quadrupole lens. In this technique, one quadrupole electrode is pulsed to prevent ions from entering the extraction zone except when an ion packet is to be extracted for mass analysis. This technique significantly reduces the noise over continuous ion injection. In the orthogonal ICP-TOFMS with pulsed-ion injection, $0.5 \mathrm{fmol}$ of analyte could be detected in $1.4 \mathrm{~ms}$ with a proper data acquisition system. Overall, the combination of a quadrupole lens and pulsed-ion injection may provide detection limits for the ICP-TOFMS that are competitive with those of quadrupole inductively coupled plasma-mass spectrometry instruments. (J Am Soc Mass Spectrom 1995, 6. 400-410)
\end{abstract}

$\mathrm{T}$ ime-of-flight mass spectrometry (TOFMS) has experienced a renaissance in the past five years, especially in its use with continuous atmospheric-pressure ion sources [1]. One of the most important aspects of instrumental development in this area is to improve the ion-transport efficiency into the TOFMS. When plasma ion sources such as the inductively coupled plasma (ICP) are used, ions from a very hot ionized gas $(\sim 6000 \mathrm{~K})$ are sampled into vacuum to form a free-jet expansion. This expansion is then skimmed to create a supersonic beam of neutrals, ions, and electrons. The ions of all masses in the beam move at roughly the same velocity, typically on the order of $7000 \mathrm{~m} / \mathrm{s}$. As a result, the ion energies in the beam are directly proportional to their masses. Also, because the ions are created in a plasma, high levels of plasma-gas ions create a beam with considerable space-charge [2].

Earlier workers employed an orthogonal TOFMS with electron impact and chemical ionization sources and demonstrated the need for a well-defined ion beam in the extraction region of the mass spectrometer

Address reprint requests to Dr. Gary $M$. Hieftje, Department of Chemistry, Indiana University, Bloomington, IN 47405.
$[3,4]$. Unfortunately, forming such a beam from ions extracted from an ICP poses a problem in ion-optic design because of the high ion flux in the beam. During the development of an inductively coupled plasma-time-of-flight mass spectrometer (ICP-TOFMS) in our laboratory we started with a simple cylindricallens system [1], which served to focus the ion beam into the extraction zone. However, in the ICP-TOFMS the extraction zone was chosen to be $2.5 \mathrm{~cm}$ in length to promote a higher duty cycle and consequently higher sensitivity. Within this extended extraction region the ion beam becomes defocused beyond the focal point. The resultant beam divergence then limits the attainable resolving power due to the higher order deviation from the optimal space focusing in a WileyMcClaren-type ion source [5]. To limit this divergence a slit was placed at the exit of the cylindrical-lens system [1]. The slit, combined with this lens system, then required a tradeoff between ion transmission and resolving power. In addition, residual divergence in the ion beam creates ion noise at the detector because ions from the continuous beam can more easily enter the flight tube during periods when the pulsed extraction field is off. 
Based on these observations in our prototype ICPTOFMS, we decided that an ion-optical setup that focuses the ions into a slitlike image would be more appropriate for coupling an ICP ion source with an orthogonal TOFMS [1]. Previous investigations of ion optics to focus ions in such an application are few. Most of the researchers involved in pulsed-extraction orthogonal TOFMS instruments have used simple cylindrical optics $[3,4,6]$ or a series of extraction skimmers or orifices [7-11] before the extraction region. Most orthogonal instruments incorporate a defining slit before the TOFMS extraction zone similar to that employed in our prototype ICP-TOFMS.

As described by Wollnik [12], electrostatic quadrupole lenses provide a method to achieve point-toparallel or astigmatic focusing of charged beams. In the case of the single quadrupole lens, ion beams are focused in one plane parallel to the optical axis and defocused in the perpendicular plane. Quadrupole multiplets offer even more efficient focusing at the focal plane but at the cost of somewhat greater complexity. In this initial investigation we employ a single electrostatic quadrupole lens to focus ions into the entrance of the orthogonal TOFMS. The performance of this ion-optical setup is compared with a simple cylindrical-lens system. In addition, it is demonstrated that the problem of continuous ion noise can be reduced by pulsing the quadrupole lens to allow ions into the extraction zone only when they will be used for mass analysis.

\section{Experimental}

\section{Time-of-Flight Mass Spectrometry and Ion Optics}

Except for the new ion optics, which will be described below, the instrumental configuration is essentially the same as described previously $[1,13]$. However, an ion reflectron now can be employed to improve resolving power (see Figure 1). Here, we describe experimental conditions and results with the use of both the linear and reflectron TOFMS. To provide increased resolving power in the reflectron TOFMS a repeller pulse of greater than $400 \mathrm{~V}$ is needed; therefore, a commercial pulse generator (DEI Model FPX 800, Fort Collins, CO) is now employed for the repeller pulsing. In addition, the differential-pumping chamber is improved such that the distance from the tip of the sampling cone to the center of the orthogonal extraction region is reduced from 50 [1] to $21.1 \mathrm{~cm}$. A better vacuum has been achieved with a cubic vacuum chamber (MDC Vacuum Products, Hayward, CA), which is actually a chamber within a chamber. In comparison to the prior system, the length of the ion-optical system has been reduced and the lens elements are easily interchangeable, which facilitates the testing of different arrangements.

In the present paper we will compare the performance of the ICP-TOFMS with two separate ionoptical arrangements. The first arrangement is a simple cylindrical-lens system similar to that used in the original TOFMS vacuum chamber $[1,13]$ (see Figure 2a). Behind the skimmer, the system consists of an extraction optic S1, which also constitutes the conductancelimiting aperture between the second and third stages. By reduction of the distance ions travel in the second stage (i.e., the skimmer-to-S1 distance, which is 12 $\mathrm{mm})$, collisions are reduced and beam throughput is increased. In the third stage, there are four lens cylinders, L1 to L4 (three of $2.61-\mathrm{cm}$ length and inner diameter of $1.58 \mathrm{~cm}$ and one of $0.32-\mathrm{cm}$ length and inner diameter of $1.27 \mathrm{~cm}$ ), and one vertical slit $(2.5 \mathrm{~mm} \times 11 \mathrm{~mm}$ ) whose long axis is parallel to the extraction region. All optical elements are separated by $0.3 \mathrm{~cm}$.

The second lens system that was investigated includes a series of cylindrical lenses followed by an electrostatic quadrupole lens (see Figure $2 b$ ). The same extraction optic $\mathrm{S} 1$ is used as in the simple cylindrical

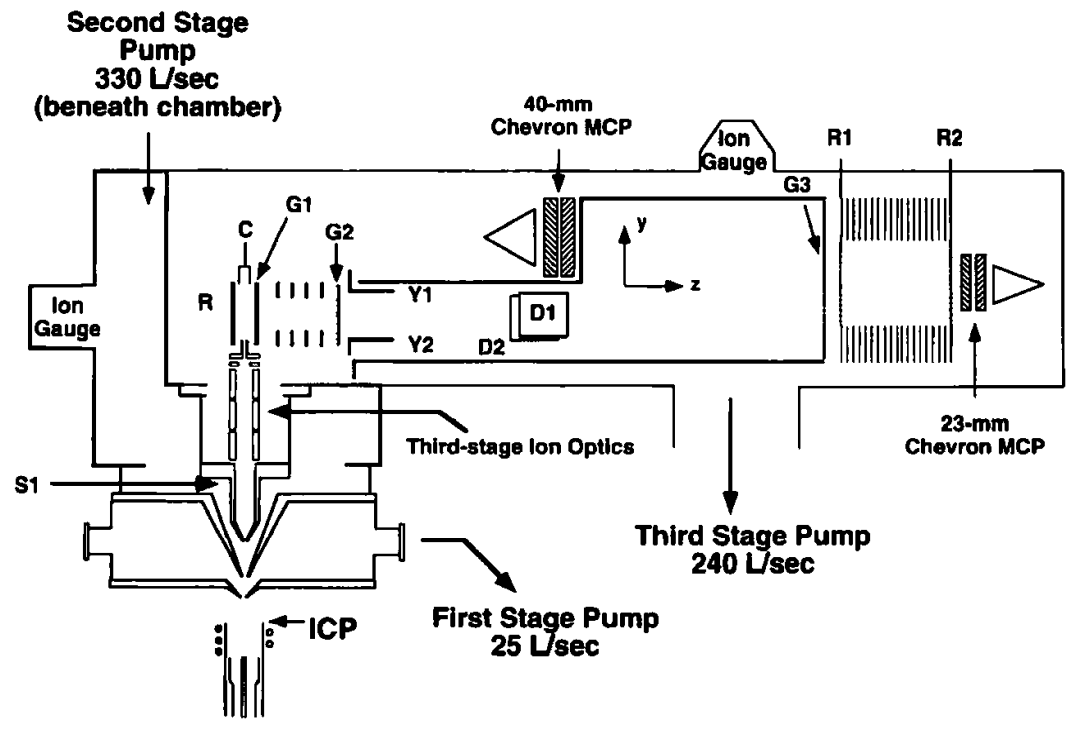

Figure 1. Layout of the ICP-TOFMS with improved vacuum chamber and ion optics. S1, second-stage extraction lens; R, repeller; $C$, Faraday cup; G1, G2, TOFMS entry grids; Y1, Y2, steering plates; D1, D2, deflection plates; G3, reflectron entrance grid; $R 1$, deceleration grid; $\mathrm{R} 2$, reflecting grid; $\mathrm{MCP}$, microchannel plate. 
lens. This element is then followed by two cylinders (the first, L1, has a length of $1.58 \mathrm{~cm}$ and inner diameter of $1.58 \mathrm{~cm}$ and the second, L2, has a length of 2.61 $\mathrm{cm}$ and inner diameter of $1.58 \mathrm{~cm}$ ). The entrance to the quadrupole lens is defined by the cylinder L3 (length of $0.3 \mathrm{~cm}$ and inner diameter of $1.27 \mathrm{~cm}$; see Figure 2b). The quadrupole consists of four circular pole surfaces (length $=2.86 \mathrm{~cm}$ ) with an open aperture $\left(2 r_{0}\right) 1.0 \mathrm{~cm}$ in diameter. The poles that defocus (elongate) the ion beam in the vertical dimension of the slit are termed $Q_{y}$, and those which focus the ions in the horizontal dimension of the slit are $Q_{x}$ poles (see Figure $2 \mathrm{~b}$ ). The exit of the quadrupole lens is defined by an element L4 that is identical to L3. Last, the same vertical slit is placed at the exit of the system as is used with the cylindrical-lens system.

Optics L1 to L4 are biased by a single power supply $(1000 \mathrm{~V}, 10 \mathrm{~mA})$ and a variable voltage-divider circuit. The two $Q_{y}$ and two $Q_{x}$ poles are connected to each other electrically for all experiments in which continuous ion injection into the TOFMS is employed. In addition, each pair of poles is biased by an independent supply (Power-One, $200 \mathrm{~V}, 150 \mathrm{~mA}$ ). During pulsed-ion injection, which will be described in detail later, the poles that are pulsed are not connected to each other electrically (i.e., one is connected to a dc potential whereas the other is pulsed). In all cases the first optic S1 is biased by a separate power supply (Keithley Instruments, Cleveland, $\mathrm{OH}$, model 244A, $2000 \mathrm{~V}, 10 \mathrm{~mA}$ ), as is the slit (Power-One, $200 \mathrm{~V}, 150$ $\mathrm{mA}$ ). Voltages applied to the TOFMS are similar to those reported earlier (see Table 1 for specific potentials); other typical ion-optic settings also can be found in Table 1. Note that most optics exhibit a range of optimum potentials that can vary on a daily basis and from chemical element to chemical element.

\section{Phosphor Screen for Ion-Beam Imaging}

To characterize the quality of the continuous ion beam within the right-angle extraction zone for each ionoptical system, we utilized a microchannel plate (MCP) and phosphor combination (see Figure 3 ). The beam image on the phosphor was photographed through a Plexiglas flange on the vacuum chamber for a variety of ion-optical conditions.

\section{Background Noise Measurements}

Noise measurements were made in the ICP-TOFMS by a gated ion-counting method. The signals were sent to a constant-fraction discriminator (CFD; Oxford Instruments, Oak Ridge, TN, model TC454) that is gated (50 ns) at the ion peak of interest. The nuclear instrumentation module pulses from the output of the CFD were counted by a rate meter (Ortec, Oak Ridge, TN, model 449). The threshold of the CFD was set to $-50 \mathrm{mV}$ in all measurements to avoid triggering on the rf noise from the ICP. All other signal measurements were
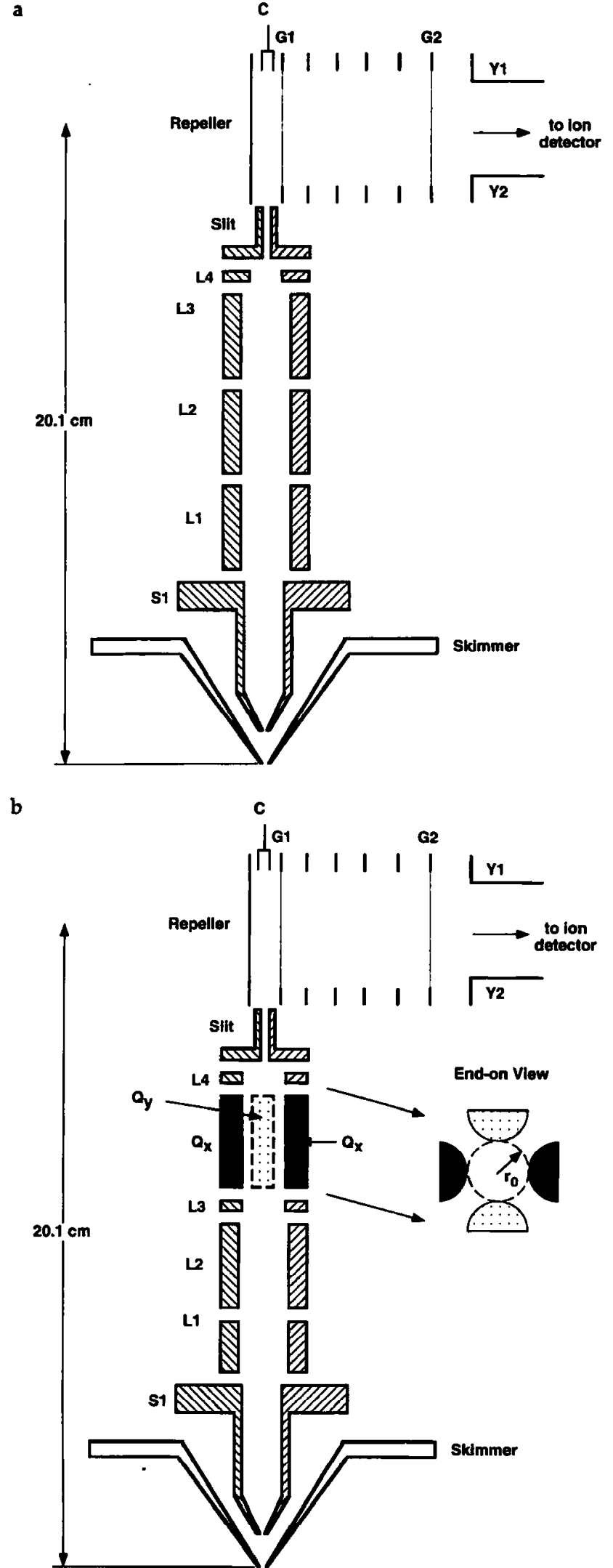

Figure 2. Diagram of both ion-optical arrangements tested for ICP-TOFMS. (a) The cylindrical-lens system (b) the quadrupolelens system. $L 1$ to $L 4$, cylinder lenses; $Q_{x}, x$ dimension poles; $Q_{y}, y$ dimension poles; $r_{0}$, radius of the quadrupole open aperture $(0.5 \mathrm{~cm})$. See Figure 1 for all other symbol definitions. 
Table 1. ICP-TOFMS operating conditions

\begin{tabular}{|c|c|c|c|}
\hline \multicolumn{4}{|c|}{ ICP } \\
\hline Forward rf power (40 MHz) & $1.35 \mathrm{~kW}(<5 \mathrm{~W}$ reflected $)$ & Central gas flow & $0.8-0.9 \mathrm{~L} / \mathrm{min}$ \\
\hline \multirow[t]{2}{*}{ Sampling depth } & $10 \mathrm{~mm}$ above the load coal & Intermediate flow & $1.0 \mathrm{~L} / \mathrm{min}$ \\
\hline & & Outer gas flow & $13.0 \mathrm{~L} / \mathrm{min}$ \\
\hline Solvent loadinga & $10.4 \mathrm{mg} / \mathrm{min}$ & & \\
\hline \multirow{2}{*}{\multicolumn{2}{|c|}{ Cylindrical lens ${ }^{c}$}} & \multirow{2}{*}{\multicolumn{2}{|c|}{ Quadrupole lens ${ }^{d}$}} \\
\hline & & & \\
\hline$\overline{\mathrm{S1}}$ & $-900 \mathrm{~V}$ & S1 & -850 to $-950 \mathrm{~V}$ \\
\hline L1 & $-360 v$ & LI & -75 to $-120 \mathrm{~V}$ \\
\hline L2 & -26 to $-60 \mathrm{~V}$ & L2 & -300 to $-350 \mathrm{~V}$ \\
\hline L3 & -50 to $-170 \mathrm{~V}$ & L.3 & -160 to $-360 \mathrm{~V}$ \\
\hline L4 & -170 to $-180 v$ & $L 4$ & -200 to $-350 \mathrm{~V}$ \\
\hline \multirow[t]{4}{*}{ Slit } & -30 to $-35 V$ & Slit & -100 to $-150 \mathrm{~V}^{\circ}$ \\
\hline & & $Q_{x}$ & +5 to $+10 \mathrm{~V}$ \\
\hline & & $a_{y}$ & -8 to $-10 v$ \\
\hline & Ir TOFMS ${ }^{\mathrm{e}}$ & \multicolumn{2}{|c|}{ Reflectron TOFMS } \\
\hline$\overline{\mathrm{G1}}$ & Ground & All same as left exce & \\
\hline $\mathbf{G} 2$ & $-2000 \mathrm{~V}$ & $\mathrm{R} 1$ & -400 to $-450 \mathrm{~V}$ \\
\hline G3, R1, R2 & $-2000 \mathrm{~V}$ & $\mathrm{R2}$ & +320 to $+340 \mathrm{~V}$ \\
\hline Flight tube & $-2000 \mathrm{~V}$ & Y2 & -2150 to $-2250 \mathrm{~V}$ \\
\hline$Y 1, D 2$ & $-2000 V$ & R pulse potential & +420 to $450 \mathrm{~V}$ \\
\hline Y2 & -2240 to $-2300 \mathrm{~V}$ & D1 pulse width & $550 \mathrm{~ns}$ \\
\hline$R$ bias potential & 0 to $-4 \mathrm{~V}$ & D1 puise delay after & $600 \mathrm{~ns}$ \\
\hline R pulse potential (at $7.1 \mathrm{kHz}$ ) & 160 to $168 \mathrm{~V}$ & repeller & \\
\hline D1 bias potential & -1960 to $-2000 \mathrm{~V}$ & & \\
\hline D1 pulse potential & $-200 V$ & & \\
\hline D1 pulse width & 310 ns & & \\
\hline $\begin{array}{l}\text { D1 pulse delay after } \\
\text { repeller }\end{array}$ & $4.00 \mu \mathrm{s}$ & & \\
\hline
\end{tabular}

${ }^{B}$ Ultrasonic nebulizer with partial desolvation.

${ }^{b}$ First stage $=0.9$ torr; second stage $=1.0 \times 10^{-4}$ torr; third stage $=2.0 \times 10^{-6}$ torr.

'See Figure $2 a$.

'See Figure $2 b$.

See Figure 1.

made with a Tektronix TDS 520 digital oscilloscope (Tektronic, Inc., Beaverton, OR) as outlined previously [1].

\section{Results and Discussion}

\section{Electrostatic Quadrupole Lens in Linear}

Time-of-Flight Mass Spectrometry

When the original cylindrical lens system was used in the ICP-TOFMS [1] we observed a tradeoff between resolving power and ion transmission. The primary reason for this tradeoff is that the circular beam image created by the cylindrical-lens system is shaped by a slit, so many of the primary-beam ions are prevented entrance to the extraction zone [1]. The point-toparallel or astigmatic focusing of the electrostatic quadrupole allows the ion beam to be focused into more of a slitlike image, which increases resolving power while maintaining the ion throughput.
For an electrostatic quadrupole lens of length $l_{\text {, }}$ aperture $2 r_{0}$, and in the case where a positive voltage is applied to the $Q_{x}$ poles and an identical negative voltage applied to the $Q_{y}$ poles, the focal lengths of the quadrupole are not the same in the $x$ and $y$ planes and are given by [11]

$$
\begin{gathered}
f_{x}=\frac{1}{k \sin (k l)} \\
f_{y}=-\frac{1}{k \sinh (k l)}
\end{gathered}
$$

where $k=\left(1 / 2 r_{0}\right) \sqrt{2 q U / m v^{2}}$ and $U$ is the voltage applied to the quadrupole elements, $m$ is the mass of the ion, $q$ is the charge on the ion, and $v$ is the velocity of the ion [11]. In the case when the voltages applied to the $Q_{x}$ and $Q_{y}$ poles are not the same, the quadrupole field is not symmetrical and the focusing properties are 


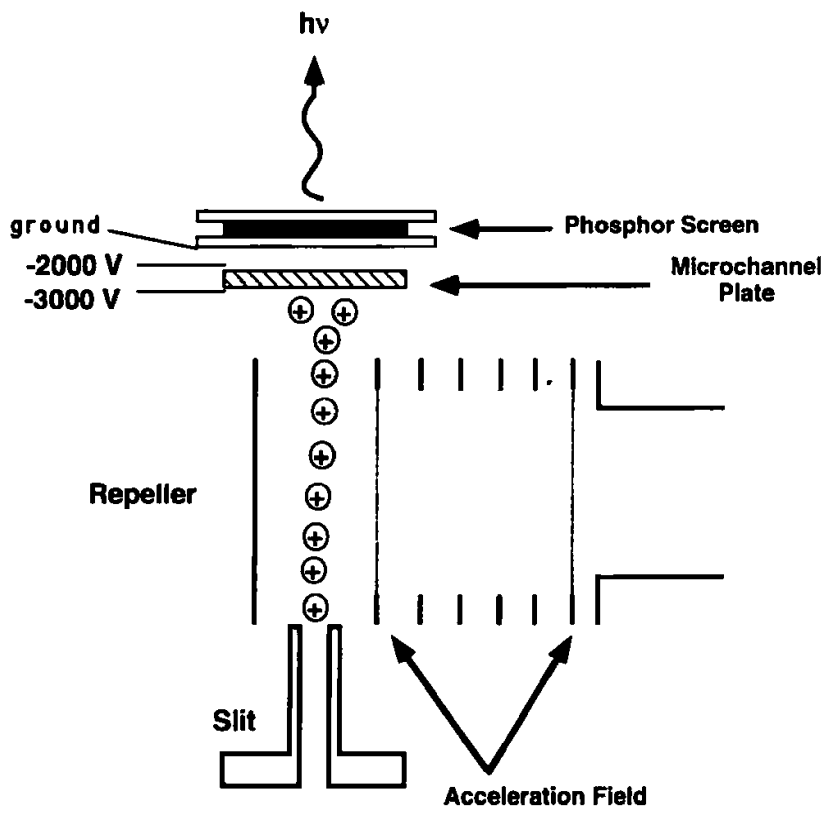

Figure 3. Microchannel plate-phosphor screen setup for ionbeam imaging.

changed accordingly. However, as long as the $Q_{x}$ poles are kept positive and the $Q_{y}$ poles negative, the ion beam is focused in the $x$ dimension and defocused in the $y$ dimension.

With the electrostatic quadrupole lens installed in the linear TOFMS instrument, we observe increased throughput and resolving power, as expected (compare spectra in Figures $4 \mathrm{a}$ and $4 \mathrm{~b}$ obtained with the cylindrical- and the quadrupole-lens systems, respectively). In particular, the spectrum in Figure $4 \mathrm{~b}$ obtained with the quadrupole lens has a higher signal for the same concentration and the resolving power is better than that with the cylindrical lens system (Figure 4a).

\section{Background Noise with Each Optical System}

A problem in our ICP-TOFMS has been the presence of background noise caused by the unintentional injection of ions [1]. As discussed in our earlier publication [1], this noise is produced by ions that leak into the flight tube from the extraction zone of the TOFMS during periods when the repeller pulse is off. This ion penetration is, not surprisingly, more significant when ions in the beam diverge in the extraction region. A comparison of the ion noise obtained with both ionoptical systems installed in the linear TOFMS can be derived from Figure 5, in which the background (measured during the nebulization of distilled/deionized $\mathrm{H}_{2} \mathrm{O}$ ) at a flight time of $22 \mu \mathrm{s}$ (which corresponds to ${ }^{133} \mathrm{Cs}$ ) is plotted as a function of the potential applied to the slit. Of course, the background noise would be roughly proportional to the square root of this total background count rate. As can be seen in Figure 5, the ion background count rate is of about the same magni-
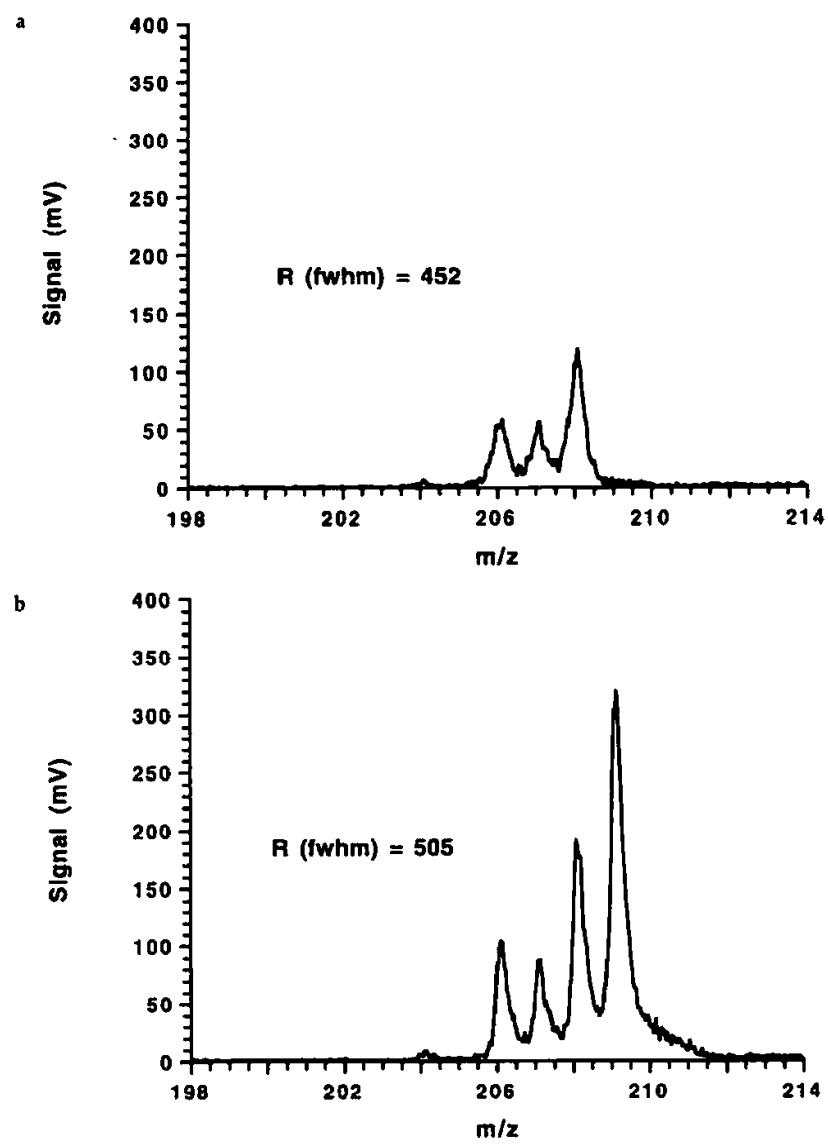

Figure 4. Mass spectra obtained with the linear TOFMS (1000shot average) for (a) the cylindrical-lens system (4-ppm Pb solution) and (b) the quadrupole-lens system (4-ppm $\mathrm{Pb}$ and $\mathrm{Bi}$ solution). $R=$ resolving power, defined at the FWHM points.

tude for both systems. This observation would indicate that the ions exhibit divergent trajectories for both lens systems. Thus, in both cases the ion beam is probably focused into the extraction region and becomes divergent while passing through the region. Nevertheless,

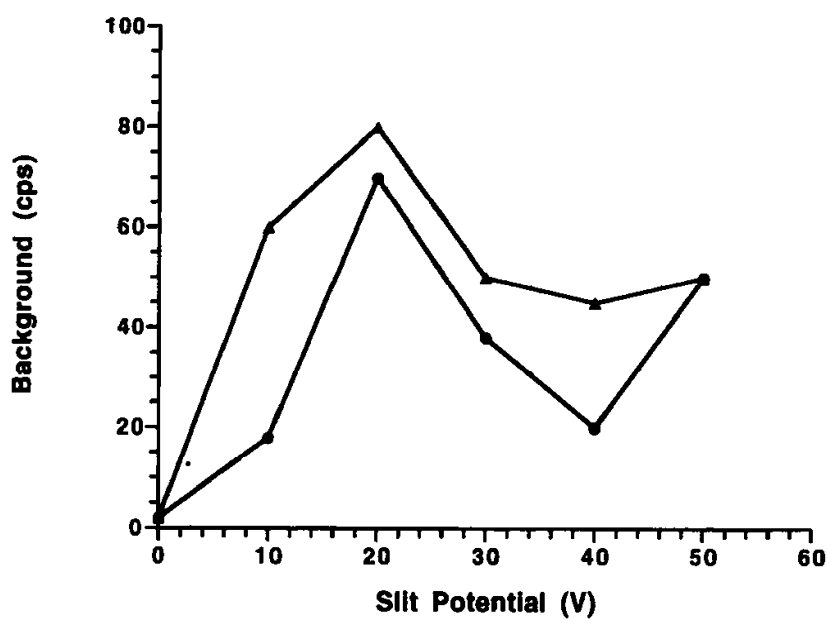

Figure 5. Background ion noise in the linear ICP-TOFMS (distilled/deionized $\mathrm{H}_{2} \mathrm{O}$ being introduced) at a flight time of $22 \mu \mathrm{s}$ (which corresponds to ${ }^{133} \mathrm{Cs}$ ) for varied slit potentials with the cylindrical-lens ( - ) and electrostatic quadrupole-lens ( - systems. 
the ion signals are higher with the quadrupole lens (Figure $4 \mathrm{~b}$ ), so the overall signal-to-noise ratio is improved. In addition, the noise levels observed with the reflectron arrangement are lower than those in the linear instrument, possibly because a majority of the scattered background ions do not have the energy to traverse the flight tube in the reflecting mode.

\section{Focusing Properties of the Direct Current Quadrupole Lens in Linear Time-of-Flight Mass Spectrometry}

As outlined above, the resolving power and ion throughput are both better with the quadrupole-lens than with the cylindrical-lens system. Also, the cylindrical lenses necessitated a tradeoff between ion transmission and resolution [1]. The quadrupole lens requires no such tradeoff (see Figure 6). As the potential on one of the cylinders preceding the quadrupole lens is adjusted, the relative signal for $\mathrm{Pb}$ and the resolving power [full width at half maximum (FWHM)] track each other quite well, unlike the earlier behavior [1]. Therefore, the need to sacrifice ion throughput for resolving power is avoided with the quadrupole lens.

The focusing abilities of the quadrupole lens can be investigated by observing changes in signal and resolving power as the potentials in the lens system are varied. The difference in potential between $Q_{x}$ and $Q_{y}$ poles will affect the transmission and focal points of the asymmetric quadrupole. Figure 7 shows how the voltage difference between the positive $Q_{x}$ poles and the negative $Q_{y}$ poles affects the resolving power and signal from a 1-ppm $\mathrm{Pb}$ solution. For these observations each pole was stepped in potential in increments of $0.5 \mathrm{~V}$. For $Q_{x}$ the increase was positive and for $Q_{y}$ the increase was negative; in other words, the effective potential difference is increased in $1.0-\mathrm{V}$ steps. As can be seen in Figure 7, as long as the appropriate potential difference between the poles is maintained, a resolving power of $\sim 700$ (FWHM) can be achieved in

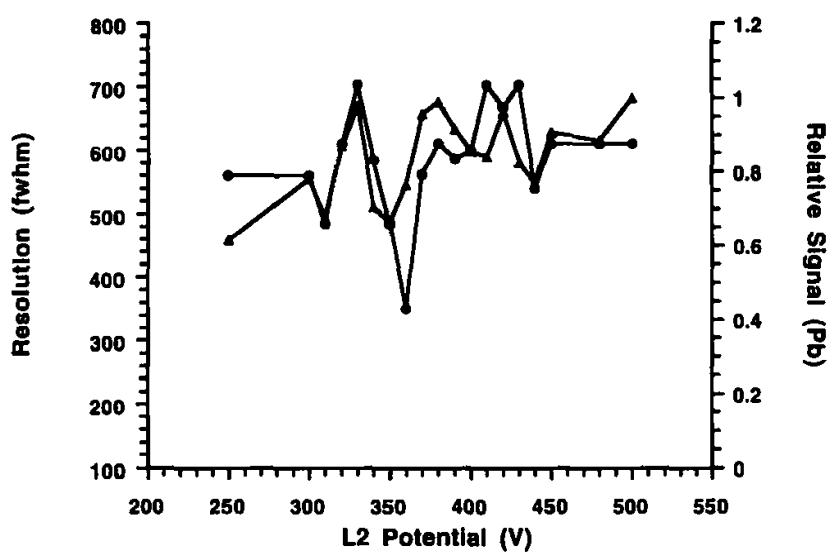

Figure 6. Effect of optic L2 potential on the relative signal (from $1 \mathrm{Ppm}{ }^{208} \mathrm{~Pb}$ solution), $\downarrow$, and resolution (FWHM), --, offered by the quadrupole lens system (see Figure $2 b$ ).

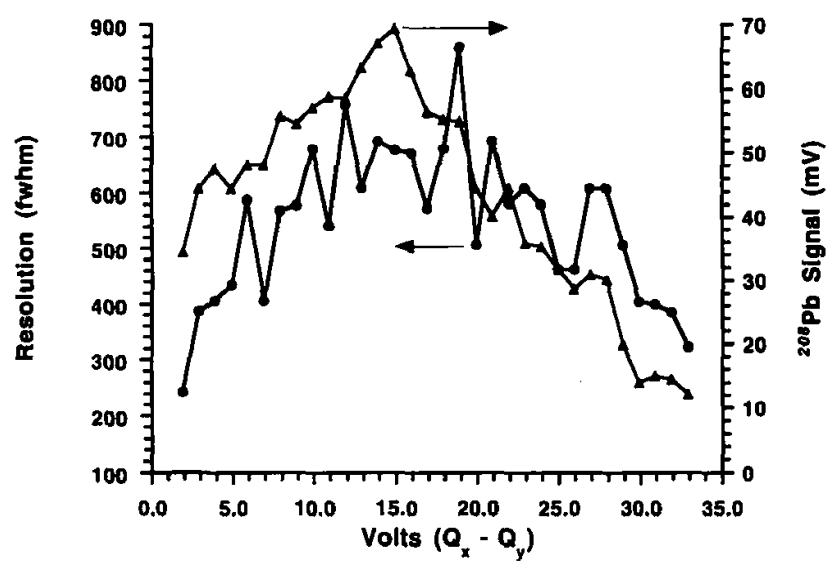

Figure 7. Effect of the potential difference between $Q_{x}$ and $Q_{y}$ poles on the signal from $1 \mathrm{ppm}{ }^{208} \mathrm{~Pb}, \star$, and on resolving power (FWHM), -

the linear TOFMS coincident with maximum ion throughput.

Application of a positive voltage to the $Q_{x}$ poles serves to focus the ion beam in the horizontal dimension of the slit. Because the spatial width of the ion beam in this dimension affects the space-focusing properties of the TOFMS [5], we would expect a higher potential on the $Q_{x}$ poles to produce a tighter focus and, consequently, greater resolving power, as long as the repeller pulse amplitude remains constant. In Figure 8 are plotted the resolving power and signal magnitude from a 1-ppm $\mathrm{Pb}$ solution as a function of the $Q_{x}$ potential while the $Q_{y}$ poles are held constant at $-8.6 \mathrm{~V}$. Significantly, there is one point (at $Q_{x}=$ $11.0 \mathrm{~V}$ ) where the resolving power (FWHM) rises to almost 1000 . Because $Q_{y}=-8.6 \mathrm{~V}$ the difference in potential between $Q_{x}$ and $Q_{y}$ is $19.6 \mathrm{~V}$, which is close to the difference in Figure 7, where resolution is at a maximum. The signal level follows a similar trend to that in Figure 7, where the higher $Q_{x}$ potentials (larger $Q_{x}-Q_{y}$ difference) yield a lower signal. Possibly the higher positive voltages on the lens move the focal

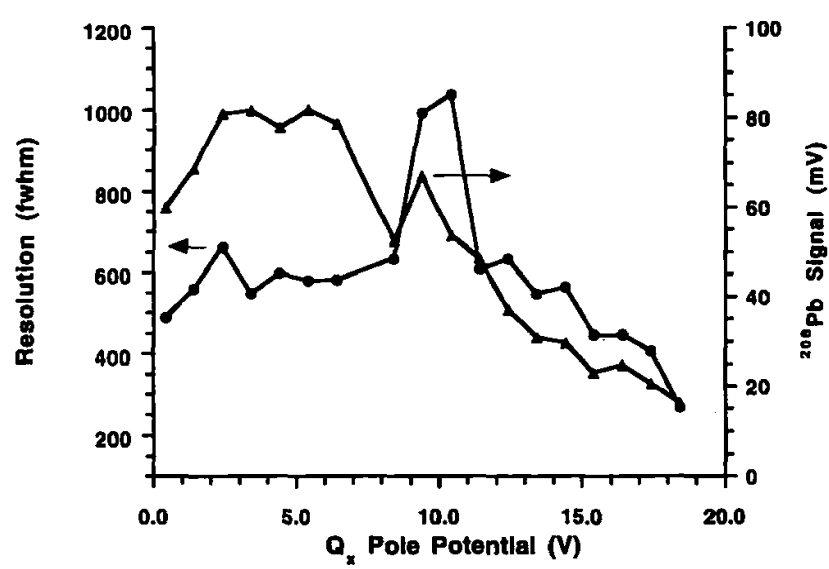

Figure 8. Effect of $Q_{x}$ pole potential (with $Q_{y}=-8.6 \mathrm{~V}$ ) on resolution (FWHM), $\leftarrow$, and on the signal from $1 \mathrm{ppm}{ }^{208} \mathrm{~Pb}, \leftarrow$. 
point in the $x$ dimension to a point where ion transmission through the slit is reduced.

\section{Phosphor Screen Diagnostics}

The MCP-phosphor screen combination shown in Figure 3 allows the direct comparison of ion-beam images from the two ion-optical systems. As expected, the cylindrical-lens system produced less intense images than did the electrostatic quadrupole. The conditions optimized for resolution in the spectrum from the cylindrical lens (see Figure 4a) produced the beam image shown in Figure 9a. This image (Figure 9a) shows that the ions are closer to the first grid (G1) than to the repeller. Not surprisingly, we observe that the background noise from scattered ions is higher when ion trajectories are closer to grid G1 (close to the flight tube); thus the signal-to-noise ratio is higher with trajectories closer to the repeller. Figure 9 a suggests a remarkably narrow beam in the extraction zone, although the curved shape no doubt compromises resolution and is probably a result of fringing fields in the extraction region. Interestingly, in the center of the image (Figure 9a) there is an oval-shaped area of lighter intensity that indicates the position of the slit. When the ion-optic potentials are off in the cylindrical-lens system, the image becomes as shown in Figure 9b. The oval-shaped region defines the exit slit and also shows the presence of neutrals and photons from the plasma.

The image for resolution-optimized conditions in the quadrupole lens that yield the spectrum in Figure $4 \mathrm{~b}$ is displayed in Figure 9c. The entire phosphor screen emits more intensely than in Figure 9a or 9b, which reflects the greater signals seen in the quadrupole-lens system. Also, the image obtained with the quadrupole lens (Figure 9c) is closer to the repeller than is the one found with the cylindrical lens (Figure 9a), so scattered-ion noise is lower. Furthermore, the optimal repeller bias for the quadrupole-lens image is only $-2.0 \mathrm{~V}$, whereas for the cylindrical lens it is -3.0 $V$, which suggests that the ion beam is more collimated with the quadrupole-lens system. This greater collimation requires less of an electric field to draw the ions toward the repeller.

When the repeller bias is set to $0.0 \mathrm{~V}$ in the quadrupole system, the ion beam is directed toward the first grid (G1) and generates the beam image in Figure $9 \mathrm{~d}$. The ion noise under these conditions observed at a flight time of $22 \mu \mathrm{s}$ (which corresponds to ${ }^{133} \mathrm{Cs}$ ) is 150 count/s, whereas that for the beam image in Figure $9 \mathrm{c}$ is only 65 counts/s. Because the ion-beam image seems to be easier to center in the extraction zone with the quadrupole lens, one might expect the

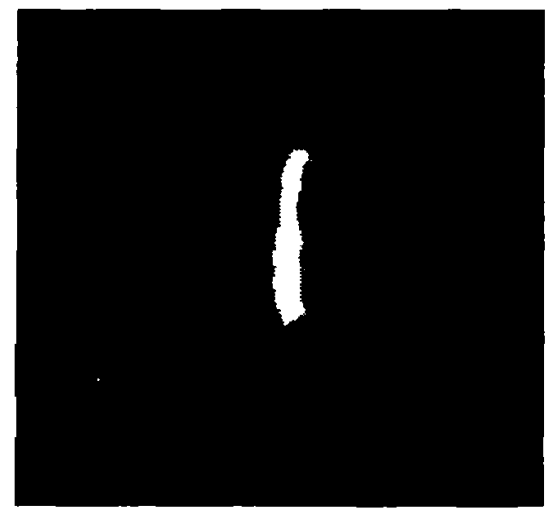

a

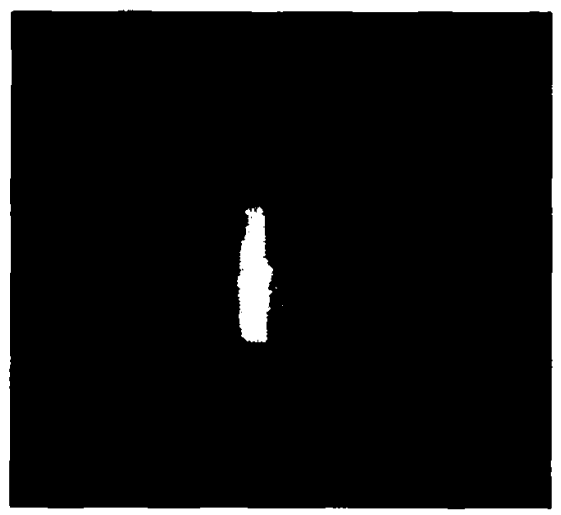

d

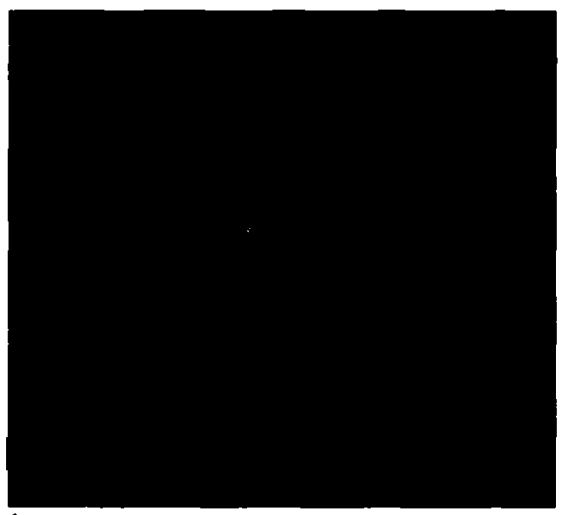

b

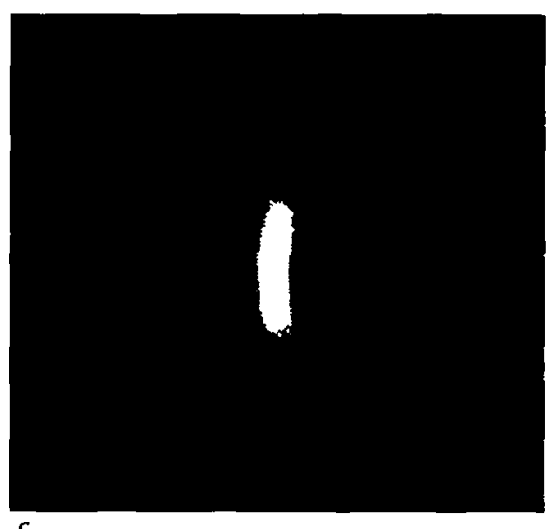

c

Figure 9. Images obtained from a phosphor screen placed at the exit of the orthogonal extraction zone (see Figure 3). (a) Image produced by cylindrical lens system under optimized conditions corresponding to the spectrum shown in Figure 4a. (b) Image produced by cylindrical system with no potentials on the optics. (c) Image produced by quadrupole lens system under optimized conditions for the spectrum shown in Figure $4 \mathrm{~b}$ (dc repeller bias $=-2.0 \mathrm{~V}$ ) and (d) image produced under the same conditions as Figure $9 \mathrm{c}$ except with dc repeller bias $=0.0 \mathrm{~V}$. 
ion noise to be lower with this system. However, the higher ion throughput offered by the quadrupole lens causes the overall noise to be roughly the same as with the cylindrical lenses.

\section{Pulsed Ion Injection}

Ions that enter the extraction zone during mass analysis of a previously injected packet are not used and serve only to create ion noise at the detector. Logically, these ions should be prevented entrance into the extraction region until they can be used. Thus, a method for beam deflection before the TOFMS entrance is needed, possibly a technique similar to that reported by workers at Bendix [11]. In these earlier studies [11], an ion-deflection plate was utilized to prevent the entrance of ions into the extraction region. In the present ICP-TOFMS instrument, ion deflection could be accomplished by pulsing one of the quadrupole poles while the other pole is maintained at a fixed dc potential. The pulse would serve to sweep ions into the right-angle extraction region and prevent their entrance when the pulse is off.

Comparison of pulsing $Q_{x}$ and $Q_{y}$ poles. To ascertain if ions could be prevented from entrance into the TOFMS extraction zone, we incremented the potential difference between the poles in each dimension to simulate the effect of pulsing each $\left(Q_{x}\right.$ and $\left.Q_{y}\right)$ pole. The ion background (proportional to the square of the background noise) was then measured at each potential

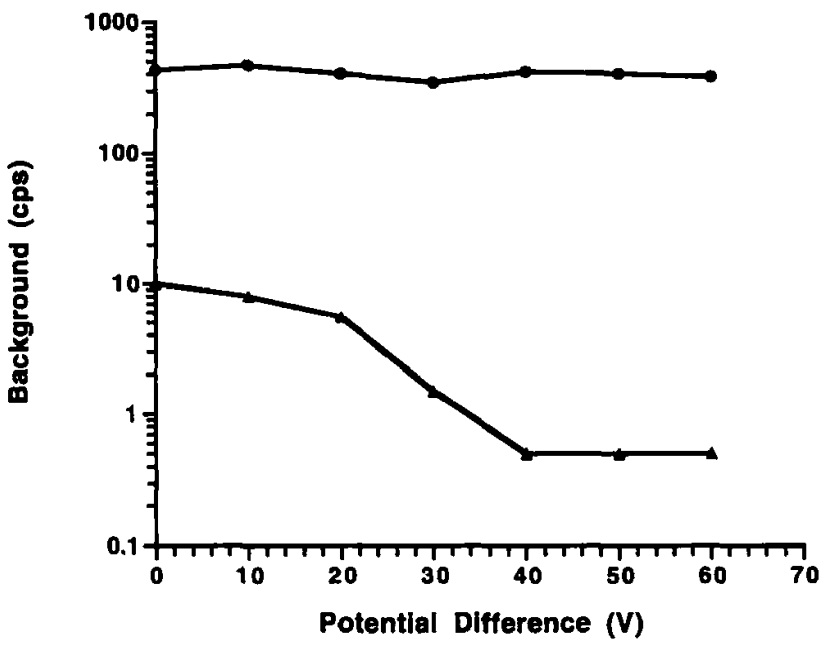

Figure 10. Effect of the potential difference between $Q_{x}$ poles, $\star$, and $Q_{y}$ poles, - , on background counts (proportional to square of the ion noise) at a flight time of $22 \mathrm{~ms}$ (corresponding to ${ }^{133} \mathrm{Cs}$ ). Notice logarithmic scale.

step (see Figure 10). Clearly, pulsing the $Q_{x}$ pole is more effective in preventing the entrance of unwanted ions into the extraction zone. This result is not surprising; the ions are being swept or deflected in the direction of the short axis $(2.5 \mathrm{~mm})$ of the slit, whereas in the $y$ dimension the ions must be deflected along the long axis $(11 \mathrm{~mm})$. A potential difference between $Q_{y}$ poles removes some noise, but does so rather inefficiently.

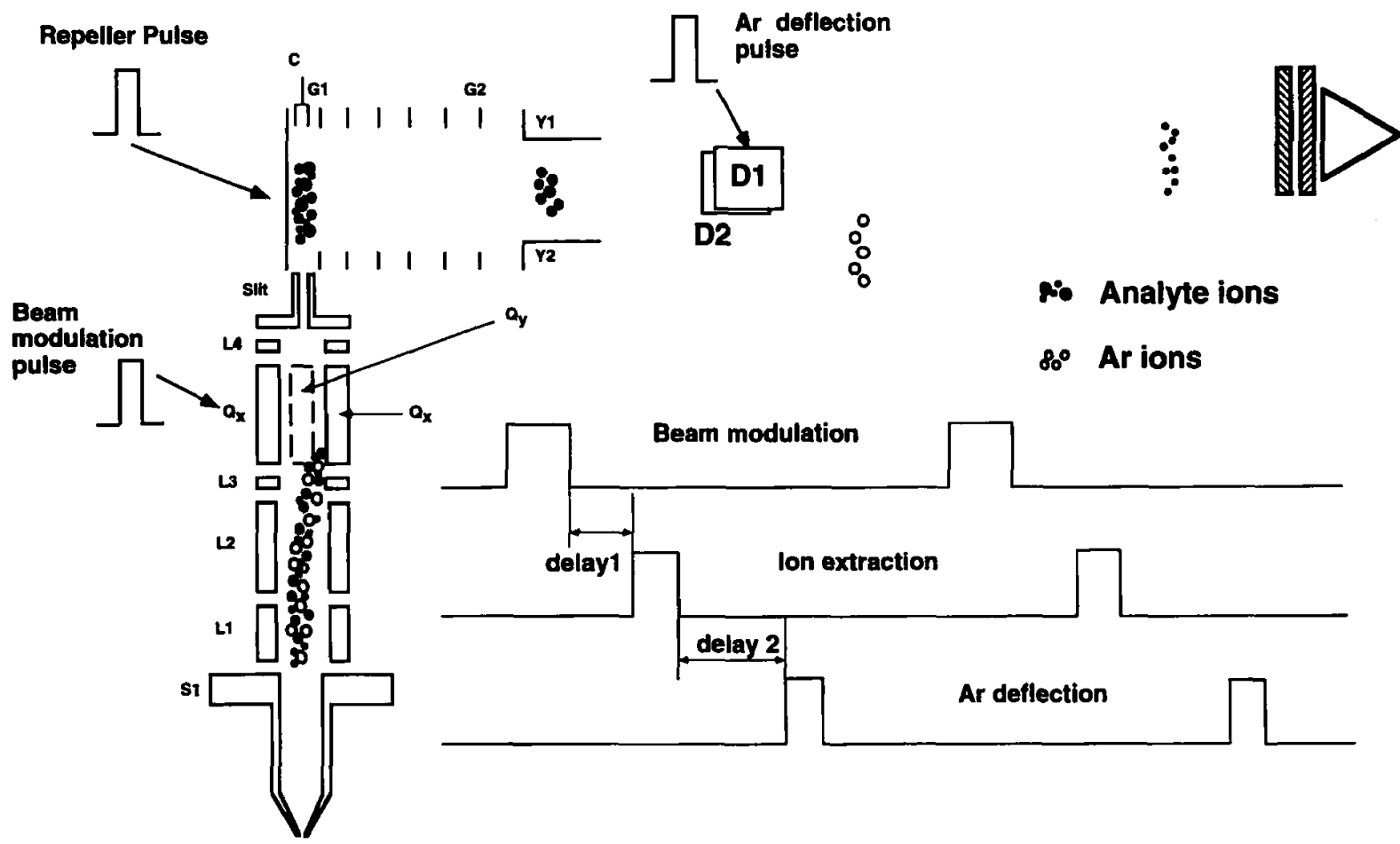

Figure 11. Schematic diagram of the pulsed ion injection process showing the beam-modulation pulse applied to the quadrupole lens and the timing among pulses in the TOFMS. See captions of Figures 1 and 2 for symbol definitions. 
Pulsed ion injection has been tested by application of pulses to both the $Q_{x}$ and $Q_{y}$ poles of the lens. Figure 11 shows how the operation is performed. A pulse of adjustable width and amplitude is applied to one pole while the other is held at dc potential. In addition, the $\mathrm{dc}$ bias of the pulse can be changed to optimize signal levels. At an adjustable delay time after the quadrupole-lens pulse, when the selected packet of ions is present within the extraction zone, the repeller pulse is applied. Somewhat later, a third pulse is applied to deflection plates D1 and D2 to deflect ${ }^{40} \mathrm{Ar}^{+}$ions from the ion packet that has been sent into the flight tube. Through variation of all ion-optic potentials, the quadrupole-pulse amplitude and width, the quadrupole-pulse bias potential, and the delay times before the repeller and deflection pulses, the signals and noise in the ICP-TOFMS instrument can be optimized. Table 2 lists typical conditions that are found to be optimal. Some of the values are expressed in the form of a range because each element optimizes at a different setting. Inadvertent changes in plasma conditions cause variation as well.

Clearly, the best situation would be one in which

Table 2. Conditions Employed During Pulsed Ion-Injection ${ }^{\text {a }}$

\begin{tabular}{lc}
\hline \multicolumn{1}{c}{ Pulsed $Q_{x}$ linear/reflectron TOFMS } \\
\hline \hline S1 & $-900 \mathrm{~V}$ \\
L1 & -450 to $-470 \mathrm{~V}$ \\
L2 & -15 to $-30 \mathrm{~V}$ \\
L3 & -40 to $-50 \mathrm{~V}$ \\
L4 & -550 to $-560 \mathrm{~V}$ \\
Slit & -100 to $-150 \mathrm{~V}$ \\
$Q_{y}$ poles & -2.0 to $-4.0 \mathrm{~V}$ \\
$Q_{x}$ (dc pole) & +4.0 to $+8.0 \mathrm{~V}$ \\
$Q_{x}$ pulse amplitude & +10 to $+25 \mathrm{~V}$ \\
$Q_{x}$ bias potential & -10 to $-20 \mathrm{~V}$ \\
$Q_{x}$ pulse width & 2 to $5 \mu \mathrm{s}$ \\
Delay after $Q_{x}$ pulse before & 9 to $10 \mu \mathrm{s}$ \\
repeller pulse & \\
\hline \hline & Pulsed $Q_{y}$ Linear/Reflectron TOFMS \\
\hline \hline S1 & -800 to $-900 \mathrm{~V}$ \\
L1 & -50 to $-70 \mathrm{~V}$ \\
L2 & -140 to $-185 \mathrm{~V}$ \\
L3 & -500 to $-600 \mathrm{~V}$ \\
L4 & -580 to $-600 \mathrm{~V}$ \\
Slit & -100 to $-150 \mathrm{~V}$ \\
$Q_{x}$ poles & +2.0 to $+5.0 \mathrm{~V}$ \\
$Q_{y}$ (dc pole) & -26 to $-50 \mathrm{~V}$ \\
$Q_{y}$ pulse amplitude & $+30 \mathrm{~V}$ to $+70 \mathrm{~V}$ \\
$Q_{y}$ bias potential & -50 to $-70 \mathrm{~V}$ \\
$Q_{y}$ pulse width & 0.8 to $1 \mu \mathrm{s}$ \\
Delay after $Q_{y}$ pulse before & \\
repeller pulse & \\
\hline & \\
\hline &
\end{tabular}

'See Table 1 for all other TOFMS conditions

See Figures 1 and 11. pulsed-ion injection would reduce noise without sacrificing the ion-signal magnitude. From the foregoing experiment, this goal would seem most likely to be met by pulsing the $Q_{x}$ poles. Spectra obtained by pulsing the $Q_{y}$ (long axis of the slit) and $Q_{x}$ (short axis of the slit) poles are shown in Figure 12a and $b$, respectively. Both spectra are for the ICP-TOFMS operated in the reflecting mode; the two spectra were obtained under the same plasma operating conditions. During these experiments, the signals were lower than had been experienced earlier because of an aged detector that exhibited a lower gain than the detector used in the linear mode. Consequently, no direct comparison can be made between the sensitivities of the two modes. Figure 12a was obtained from a 4-ppm solution of $\mathrm{Pb}$ and $\mathrm{Bi}$ and represents the average of 1000 shots (input ion pulses). The sensitivity in the case of $Q_{y}$ pulsing is approximately $24 \mathrm{mV} / \mathrm{ppm}$. If the background noise $\left(N_{\mathrm{b}}\right)$ is defined as the standard deviation of the background in a time window $100 \mathrm{~ns}$ long or 50 points from 50.242 to $50.342 \mu \mathrm{s}$, one obtains a $S / N_{\mathrm{b}} \sim$ 103. With a pulsed $Q_{x}$ pole a 40 -ppm solution of both $\mathrm{Pb}$ and $\mathrm{Bi}$ produced the spectrum in Figure $12 \mathrm{~b}$ (again,
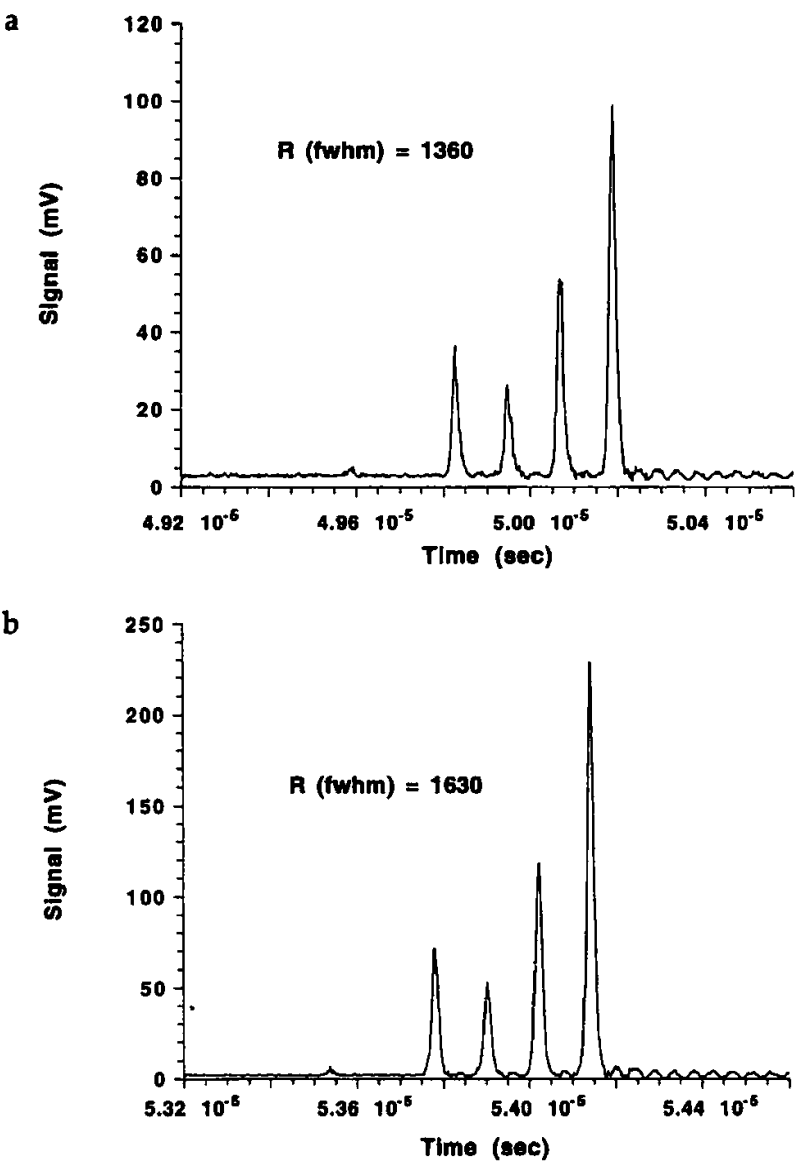

Figure 12. Time-of-flight mass spectra obtained with the reflectron ICP-TOFMS (1000-shot average) for pulsed ion injection utilizing (a) the $Q_{y}$ poles (4 ppm solution of $\mathrm{Pb}$ and $\mathrm{Bi}$ ) and $(\mathrm{b})$ $Q_{x}$ poles (40 ppm solution of $\mathrm{Pb}$ and $\mathrm{Bi}$ ). See Table 2 for operating conditions. $R=$ resolving power defined at fwhm. 
1000 input pulses) in which the sensitivity is approximately $5.7 \mathrm{mV} / \mathrm{ppm}$ and where the $S / N_{\mathrm{b}}$ is $111\left(N_{\mathrm{b}}\right.$ determined from 54.196 to $54.296 \mu \mathrm{s}$ ). Unfortunately, most of the noise contribution to the $S / N_{b}$ values is from the ringing seen in both spectra after the ${ }^{209} \mathrm{Bi}$ signal. Because this ringing is approximately proportional to the signal magnitude, it is not a surprise that the $S / N_{\mathrm{b}}$ values are comparable. Better guidance in the estimation of detection capabilities can be obtained by a comparison of background levels, which are obviously greater in Figure 12a $\left(Q_{y}\right.$ pole pulsing) than in Figure $12 b\left(Q_{x}\right.$ pole pulsing). When gated ion counting is used, the background level is measured to be 15 count/s at a flight time of $40 \mu \mathrm{s}\left({ }^{133} \mathrm{Cs}\right)$ when the $Q_{y}$ pole is pulsed, but only 1 count/s when the $Q_{x}$ pole is pulsed.

Pulsing either pole results in excellent resolving power, but the $Q_{x}$ pulsing yields the higher value of 1630. It seems that sweeping the beam across the slit discriminates against certain ion energies, thereby narrowing the energy distribution in the dimension of the sweeping. The $Q_{y}$ pulsing will not affect energies in the direction of the ion flight path in the TOFMS; therefore, $Q_{x}$ pulsing achieves better resolving power because it generates a narrower kinetic-energy distribution along the flight-tube axis.

Overall, pulsed-ion injection works well with the quadrupole lens. Pulsing the $Q_{y}$ pole seems to offer higher ion throughput than does pulsing the $Q_{x}$ pole, but the background level with a pulsed $Q_{x}$ pole is an order of magnitude lower and resolving power is slightly higher.

Pulsed versus continuous ion injection. Figure 13a and $b$ compare spectra obtained with the linear TOFMS for continuous and pulsed ion injection $\left(Q_{x}\right.$ poles pulsed in Figure 13b), respectively. Both spectra are for a 100-ppb Bi solution. The detector utilized in these experiments was a new chevron microchannel assembly that offered considerably higher signals than the detector used to generate Figure $4 a$ and $b$. The continuous ion-injection spectrum (Figure 13a) is an average of 100 shots. The noise is defined here in a region of $100 \mathrm{~ns}$ (mean and standard deviation of 50 points) from 28.4 to $28.5 \mu \mathrm{s}$, and the signal is taken as the amplitude at $28.1 \mu \mathrm{s}$ (which corresponds to ${ }^{209} \mathrm{Bi}^{+}$) minus the mean background level. The signal-to-noise ratio $\left(S / N_{\mathrm{b}}\right)$ in this case is 13 , which corresponds to a detection limit of $25 \mathrm{ppb}$. For Figure 13b, which was obtained during pulsed ion injection and with only 10 input shots being averaged, $\left(S / N_{b}\right)=94$. This $\left(S / N_{b}\right)$ yields an approximate detection limit of $3 \mathrm{ppb}$.

It is highly significant that the signal amplitude is greater in Figure 13b than in Figure 13a even though the number of averaged spectra is 10 times lower. The greater number of averages that were acquired to produce Figure 13a served to reduce not only the noise level, but also the signal amplitude; because individual single-shot spectra contain signals for bismuth over
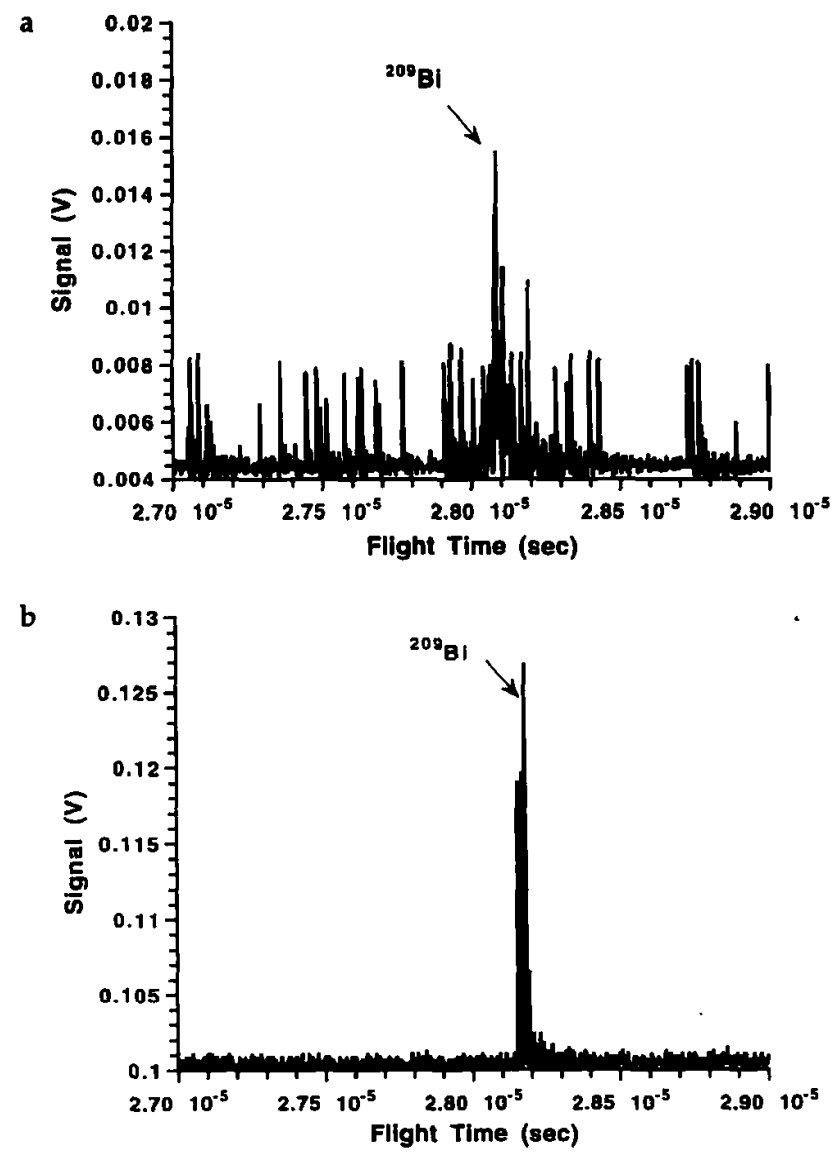

Figure 13. Time-of-flight mass spectra obtained with the linear TOFMS for (a) continuous ion injection $(100 \mathrm{ppb} \mathrm{Bi}, 100$-shot average) and (b) pulsed ion injection using $Q_{x}$ poles ( $100 \mathrm{ppb} \mathrm{Bi}$, 10 shot average). See Table 2 for operating conditions.

a distribution of flight times, the integrated signal amplitude at a given point (flight time) does not rise linearly with the number of accumulated spectra. Moreover, the average signal value actually drops with time because of the longer averaging process.

It is instructive to consider the total time required to take the signal measurement in Figure $13 \mathrm{~b}$ and the amount of analyte material that is consumed during this interval. The length of time required to generate 10 spectra is only $1.4 \mathrm{~ms}$ (although the current digital oscilloscope consumes about $1 \mathrm{~s}$ of measurement time to sum the spectra because of its limited signalintegration rate). With a sample-solution uptake rate of $1.5 \mathrm{~mL} / \mathrm{min}$, the total volume of solution being analyzed is $0.035 \mu \mathrm{L}$ ( $35 \mathrm{~nL}$ ). The concentration of $\mathrm{Bi}$ in this solution is $100 \mathrm{ppb}$, so the amount of Bi detected in this time period is $3.5 \times 10^{-12} \mathrm{~g}$ or $17 \mathrm{fmol}$, and the $\left(S / N_{b}\right)$ ratio is approximately 94 . Therefore, the minimum detectable amount $\left(S / N_{b}=3\right)$ is $0.5 \mathrm{fmol}$ or 100 $\mathrm{fg}(0.1 \mathrm{pg})$. These detection capabilities are equal to or better than has been achieved by others who used orthogonal TOFMS instruments and have reported femtomole detection limits for molecular species in roughly the same time period $[7,8]$. 


\section{Conclusions}

An electrostatic quadrupole-lens system has been investigated as an alternative to a simple cylindrical-lens system in an orthogonal ICP-TOFMS. The singlet quadrupole lens offers the ability to focus ions into a slitlike image, which is more conducive to improved ion throughput and resolving power in the orthogonal TOFMS. Also, the tradeoff between sensitivity and resolution required by the cylindrical lenses [1] is not necessary with the quadrupole lens. The background noise generated by scattered ions is nearly identical in both ion-optical systems; however, the quadrupole lens offers the ability to remove the unwanted ion noise through a technique called pulsed-ion injection, where only those ions used for mass analysis are permitted to enter the extraction zone of the TOFMS. In the future, the incorporation of a quadrupole doublet could further improve the ion transmission of the lens system.

With pulsed-ion injection the $S / N_{\mathrm{b}}$ can be improved by a factor of 10 with no loss in resolving power. Moreover, because of the speed of the TOFMS and the increase in $S / N_{\mathrm{b}}$, approximately $0.5 \mathrm{fmol}$ of bismuth $(0.1 \mathrm{pg})$ could be detected in $1.4 \mathrm{~ms}$, provided the electronic detection system responds at the spectral generation rate of $7 \mathrm{kHz}$. These results suggest that with an appropriate integrating detection scheme the detection limits of the ICP-TOFMS should compete effectively with the quadrupole ICP-mass spectrometry instruments currently available.

\section{Acknowledgments}

This research was funded in part by the National Institutes of Health through grant R01 GM48653.

\section{References}

1. Myers, D. P.; Li, G.; Yang, P.; Hieftje, G. M. J. Am. Soc. Mass Spectrom., 1994, 5, 1008-1016.

2. Tanner, S. D. Spectrochim. Actn 1992, 47B, 809-823.

3. Dawson, J. H. J.; Guilhaus, M. Rapid. Commun. Mass Spectrom. 1989, 3, 155-159.

4. Coles, J.; Guilhaus, M. Trends Anal. Chem. 1993, 12, 203-213.

5. Wiley, W.C.; McClaren, I. H. Rev. Sci. Instrum. 1955, 26, 1150-1157.

6. Michael, S. M.; Chien, B. M.; Lubman, D. M. Anal. Chem. 1993, 65, 2614-2620.

7. Verentchikov, A. N.; Ens, W.; Standing, K. G. Anal. Chem. 1994, 66, 126-133.

8. Boyle, J. G.; Whitehouse, C. M. Anal. Chem. 1992, 64, 2084-2089.

9. Sin, C.H.; Lee, E.D.; Anal. Chem. 1991, 63, 2897-2900.

10. Dodonov, A. F.; Chernushevich, I. V.; Laiko, V. V. Book of Abstracts from the 12th International Mass Spectrometry Conference; Amsterdam, Holland, August 1991, p 153.

11. O'Halloran, G. J.; Walker, L. W. Technical Document ASD TDR 62-644, Part I and II; prepared under contract AF 33(657)-11018; Bendix Corporation, Research Laboratories Division: Southfield, MI, November 1964.

12. Wollnik, H. Optics of Charged Particles; Academic Press: New York, 1987; pp 48-88.

13. Myers, D. P.; Heintz, M. J.; Mahoney, P. P.; Li, G.; Hieftje, G. M. Appl. Spectrosc. 1994, 48, 1337-1346. 